\title{
The X-ray structure of L-threonine dehydrogenase from the common hospital pathogen Clostridium difficile.
}

\author{
Eyram Adjogatse, ${ }^{a}$ Josh Bennett,${ }^{a}$ Jingxu Guo,${ }^{a, b}$ Peter \\ T. Erskine,,$^{a, c}$ Steve P. Wood, ${ }^{a, d}$ Brendan Wren ${ }^{e}$ and Jonathan \\ BARTHOLOMEW COOPER ${ }^{a, c *}$ \\ ${ }^{a}$ Division of Medicine, UCL, Gower Street, London, WC1E 6BT, England, \\ ${ }^{b}$ The Department of Medicine, University of Cambridge School of Clinical \\ Medicine, Cambridge, CB2 0QQ, England, ${ }^{c}$ Department of Biological Sciences, \\ Birkbeck, University of London, Malet Street, Bloomsbury, London, WC1E \\ 7HX, England, 'Institute of Biomedical and Biomolecular Science, School of \\ Biological Sciences, University of Portsmouth, King Henry Building, \\ Portsmouth, PO1 2DY, England, and ${ }^{e}$ London School of Hygiene and Tropical \\ Medicine, Keppel Street, London, WC1E 7HT, England. \\ E-mail: jon.cooper@ucl.ac.uk
}

\begin{abstract}
In many prokaryotes, the first step of threonine metabolism is catalysed by the enzyme threonine dehydrogenase $(\mathrm{TDH})$ which uses $\mathrm{NAD}^{+}$to oxidise its substrate to 2-amino-3-keto-butyrate. The absence of a functional TDH gene in humans suggests that inhibitors of the enzyme may have therapeutic potential against pathogens which are reliant on this enzyme. We have cloned and over-expressed TDH from Clostridium difficile and have determined the X-ray structures of the apoenzyme form at $2.6 \AA$ resolution.
\end{abstract}

\section{Introduction}

There are three known metabolic pathways of threonine catabolism, the initial steps of which are catalysed by L-threonine aldolase (EC 4.1.2.5), L-threonine dehydratase (also known as L-threonine deaminase; EC 4.2.1.16) and L-threonine 3-dehydrogenase (TDH; EC 1.1.1.103), the latter being the dominant pathway in many species (Marcus \& Dekker, 1993; Edgar, 2002). The activity of threonine aldolase has been shown to be low or insignificant in prokaryotes (Lam et al., 1980) and eukaryotes (Bird \& Nunn, 1983), and no expression of the enzyme has been detected in humans (Edgar, 2005). In contrast, the pathway initiated by TDH is significant in many prokaryotes (Marcus \& Dekker, 1993; Edgar, 2002)

PREPRINT: Acta Crystallographica Section F

A Journal of the International Union of Crystallography 
and becomes dominant in certain metabolic states in animals (Bird \& Nunn, 1983). TDH is a mitochondrial matrix enzyme which uses $\mathrm{NAD}^{+}$to oxidise threonine to 2-amino-3-keto-butyrate (AKB) (Fig. 1). The second enzyme in this pathway, 2-amino-3-keto-butyrate ligase (KBL, Schmidt et al., 2001), uses coenzyme A to convert AKB to glycine and acetyl coenzyme A (Ac-CoA) (Fig. 1). This pathway plays a variety of roles in both prokaryotes and eukaryotes, including energy production, homeostasis and fatty-acid synthesis (Millerioux et al., 2013; Mazet et al., 2013).

Dehydrogenases are divided into short-, medium- and long-chain reductases (SDRs, MDRs and LDRs, respectively) (Kavanagh et al., 2008). TDHs have been found in the MDR family and these are tetrameric, having a requirement for divalent metal cations, such as zinc (Boylan \& Dekker, 1978, 1981; Bowyer et al., 2009; Higashi et al., 2005; Ishikawa et al., 2007; Machielsen \& van der Oost, 2006). Other TDHs are found in the SDR family and are dimeric with no requirement for metal ions (Yoneda et al., 2010, 2012; Ueatrongchit \& Asano, 2011). They also have sequence and structural similarity with UDP-galactose 4'-epimerase (UDP-GalE).

The Ac-CoA produced by the TDH of the sleeping sickness parasite T. brucei was shown to be of comparable importance for fatty-acid synthesis as that produced by pyruvate dehydrogenase and inhibition of both enzymes is lethal for the parasite (Millerioux et al., 2013; Mazet et al., 2013). In addition, the glycine produced by the parasite TDH is incorporated into trypanothione, an essential antioxidant. It has long been known that inhibition of the parasite TDH by tetraethylthiuram disulfide (better known as the alcohol-aversion drug antabuse) has trypanocidal effects (Cross et al.,1975; Linstead et al., 1977). The absence of functional TDH in the human genome (Edgar, 2002) suggested that its reliance on TDH may be an important achilles heel for the parasite and other pathogens. This led us to express and crystallise the TDH enzymes from a range of pathogenic species, including $T$. brucei for structure analysis and fragment screening (Adjogatse et al., 2018).

In recent decades, the spore-forming bacterium Clostridium difficile has emerged as a major cause of hospital-acquired infections of the colon that are associated with prolonged antibiotic treatment and are increasing in mortality (Banawas, 2018). The rapid metabolism of threonine, and several other amino acids, by clostridia suggested that the TDH from $C$. difficile is also of interest as a potential candidate for structure-based drug design, although clostridia appear capable of using all three alternative pathways of threonine metabolism (Fonknechten et al., 2010; Neumann-Schaal et al., 2019). To this end we have cloned and overexpressed the TDH from $C$. difficile and have determined the X-ray structures of the apoenzyme form at $2.6 \AA$ resolution. 


\section{Methods}

\subsection{Cloning, expression and purification}

The gene for the 318 amino acid SDR TDH from $C$. difficile (UniProt ID: A0A4U8WM48_CLODI) was amplified from genomic DNA of strain $630 \Delta$ erm by PCR using KOD polymerase (Novagen) with forward and reverse primers which were designed to introduce cleavage sites for the restriction enzymes NdeI and BamHI and to convert the start codon from TTG to ATG:

Forward: gcgectggatctagacatATGaaaaaaatacttataacaggtgc

Reverse: gcgcaagcttggatccgccctatttacctattcctttttctg

Following digestion of the amplified DNA with NdeI and BamHI, the TDH gene was ligated with the expression vector pET16b using standard methods and transformed into $E$. coli strains DH5 $\alpha$ (for confirmatory sequencing) and BL21(DE3) for expression of the deca-His-tagged enzyme. Primer synthesis and plasmid sequencing were conducted by Yorkshire Bioscience, UK.

In order to obtain soluble $C$. difficile TDH enzyme, two steps were necessary. Firstly, the cells were grown to mid-log phase at 310 Kelvin and then given a 25 min heat shock at $315 \mathrm{~K}$ followed by cooling on ice for 5 min prior to induction with IPTG $(0.3 \mathrm{mM})$ and shaking at $289 \mathrm{~K}$ for 2 days. Secondly, during nickel affinity purification of the enzyme from the sonicated cell supernatant, the TDH was eluted from the nickel column using $0.5 \mathrm{M}$ imidazole in a single step into 50 $\mathrm{mM}$ Tris- $\mathrm{HCl}$ buffer at $\mathrm{pH} 7.5$ containing $1 \mathrm{mM} \beta$-mercaptoethanol, $0.1 \mathrm{M} \mathrm{NaCl}$ and $10 \%$ glycerol. The enzyme was further equilibrated with the same buffer by centrifugal ultra-filtration to remove the imidazole elutant and concentrate the enzyme for crystal screening and storage at $253 \mathrm{~K}$. The catalytic activity was confirmed by mixing $1 \mu \mathrm{L}$ enzyme with $25 \mu \mathrm{L}$ of solution containing 50 $\mathrm{mM}$ threonine, $2 \mathrm{mM} \mathrm{NAD}^{+}$and $200 \mathrm{mM}$ Tris- $\mathrm{HCl} \mathrm{pH} \mathrm{8.4,} \mathrm{and} \mathrm{following} \mathrm{the}$ decrease in absorbance at $340 \mathrm{~nm}$ with a Nanodrop ND-1000 spectrophotometer, although the kinetic parameters of the enzyme were not determined.

\subsection{Crystallisation}

Screening for crystallisation conditions was undertaken by the vapour diffusion method using 24-well hanging drop plates into which $0.7 \mathrm{ml}$ of each of the

Molecular Dimensions Structure Screens I and II were pipetted. Siliconised glass cover-slips, on which $5 \mu \mathrm{L}$ of $C$. difficile $\mathrm{TDH}$ at a concentration of $5 \mathrm{mg} / \mathrm{ml}$ were mixed with an equal volume of well solution, were inverted and sealed above the wells with high vacuum grease (Dow Corning). The trays were left at $293 \mathrm{~K}$ and, after several days, large crystals appeared in $0.2 \mathrm{M}$ lithium sulphate, 0.1 M Tris-HCl pH 8.5, 30 \% v/v PEG 4000 (Structure Screen I, condition 35). Subsequently crystals were found to grow in this condition with PEG 4000 in

IUCr macros version 2.1.11: 2020/04/29 
the concentration range $20-35 \% \mathrm{v} / \mathrm{v}$.

\subsection{X-ray data collection and structure analysis}

Crystals were transferred to $10 \mu \mathrm{L}$ droplets of well solution using a cryoloop (Molecular Dimensions) and four $1 \mu \mathrm{L}$ droplets of glycerol were stirred in slowly to cryoprotect the crystals prior to freezing in liquid nitrogen. Data were collected from the crystals at $100 \mathrm{~K}$ at Diamond Light Source (DLS) beamline I04-1 using a PILATUS $2 \mathrm{M}$ detector and a wavelength of $0.92 \AA$. A total of $190^{\circ}$ of data were collected using a rotation range of $1^{\circ}$ and an exposure time of 4 s per image.

The diffraction data were processed automatically at the beamline using MOSFLM (Battye et al., 2011) which suggested that the crystal was of the trigonal point group 321 and the systematic absences along $l$ indicated that the space group was either $P 3_{1} 21$ or $P 3_{2} 21$. Scaling and merging of the data with Pointless (Evans \& Murshudov, 2013) followed by molecular replacement using MolRep (Vagin \& Teplyakov, 2010) with a monomer of T. brucei TDH (Adjogatse et $a l ., 2018$ ) as the search model indicated that the correct choice of space group was $P 3_{1} 21$. The crystals had unit cell dimensions of $a, b=180.87 \AA, c=88.35$ $\AA, \alpha, \beta=90.0^{\circ}, \gamma=120.0^{\circ}$ and possessed 2 molecules per asymmetric unit with a high solvent content of $78.9 \%$ (Kantardjieff \& Rupp, 2003). The structure was rebuilt using Coot (Emsley et al., 2010) and refined using REFMAC (Murshudov et al., 1997; 2011), both in the CCP4 suite (Winn et al., 2011). This process also included one round of simulated annealing torsional molecular dynamics at $5000 \mathrm{~K}$ which was performed using CNS (Brünger et al., 1997). Data processing and refinement statistics are shown in Table 1. Figures of the structure were prepared using CueMol (http://www.cuemol.org/en).

\section{Results}

The structure of TDH from $C$. difficile has been determined at a resolution $2.6 \AA$ and refined to an R-factor of $20.0 \%$ and an R-free of $23.9 \%$ (Table 1 ). The tertiary structure is shown in Fig. 2. According to DynaRama (Casañal et al., 2020) $85.8 \%$ of the amino acids are in the most favoured region of the Ramachandran plot.

A sequence alignment of $C$. difficile TDH with the enzymes from $F$. frigidimaris, T. volcanum, mouse M. musculus and T. brucei is shown in Fig. 3. These short chain TDH enzymes have sequence identities of $48 \%, 45 \%, 41 \%$ and 39 $\%$ with the $C$. difficile enzyme.

The enzyme consists of a Rossmann fold domain with some extensive elaborations (predominantly at the C-terminal end of the protein) which create the catalytic domain (Fig. 4). The first main elaboration follows the Rossmann fold 
strand $\beta 6$ and consists of $\beta 7, \alpha 7, \beta 8$ and $\beta 9$. The next two secondary structure elements, $\alpha 8$ and $\beta 10$, appear to form part of the Rossmann fold domain while the remainder of the protein forms the bulk of the catalytic domain. This domain is substantially helical but contains two small sheet regions, the first of which is parallel and is formed by $\beta 8$ and $\beta 12$ while the second one is formed by $\beta 7, \beta 9$ and $\beta 11$ with mixed topology.

The $C$. difficile TDH monomer superimposes on the the $T$. brucei enzyme with an RMSD C $\alpha$ deviation of $1.42 \AA$ for 307 structurally equivalent residues (Fig. 5). The dimers of these two enzymes superimpose with an RMSD of 1.71 $\AA$ demonstrating the strong conservation of quaternary structure (Fig. 6a). Use of the EBI PISA server (Krissinel \& Henrick, 2007) reveals that the dimer interface of the $C$. difficile enzyme has a buried surface area of $1275 \AA^{2}$ per monomer and an estimated interaction energy of $-13.5 \mathrm{kcal}$ per mol. The interface occurs between the Rossmann fold domains and involves 15 hydrogen bonds and 8 saltbridges. The contacts are predominantly formed by helices $\alpha 5$ and $\alpha 6$ from both subunits, which are the largest helices in the molecule (Fig. 3) and show a high degree of conservation (Fig. 6b).

The electrostatic surface of the dimer shows pronounced electropositive cavities which form the binding site for the phosphodiester of the NAD cofactor (Fig. $6 c)$. The NAD cofactor and the competitive inhibitor pyruvate present in the $T$. brucei complex (RCSB-ID: 5lc1) were used to indicate the position of the active site residues (Fig. 7). Four residues making hydrogen bonds with the modelled ligands were identified: Ser 80, Ser 117, Thr 184 and Tyr 142. Of these Ser 80 and Thr 184 are invariant (Fig. 3), Tyr 142 can be Phe and Ser 117 is conservatively replaced by Thr in some TDH's. The reaction involves the abstraction of protons from the substrate $\mathrm{C} \beta$ carbon atom by the nicotinamide cofactor and from the substrate hydroxyl group by a basic enzyme residue. On the basis of other structures, it has been speculated that the conserved residues Tyr 142 and Ser/Thr 117 could perform this role (Adjogatse et al., 2018; Yoneda et al., 2012). This tyrosine residue is part of a YGxxK motif (Fig. 3) which includes an invariant lysine that has been hypothesised to play a part in the transfer of the proton (e.g. Adjogatse et al., 2017), although in the C. difficile apo-TDH structure, the sidechain of this residue (Lys 146) points away from the predicted substrate binding site.

The loop between Thr 177 and Thr 183 immediately precedes the active site residue Thr 184 and is rich in the flexible amino acid glycine. This loop is shown on the lower-right of Fig. 7 close to the predicted position of the inhibitor pyruvate. In the T. brucei enzyme, this loop was found to occupy two positions depending on whether cofactor was bound or not (Adjogatse et al., 2018). Comparison of the two structures demonstrates that this loop is in the open conformation in $C$. difficile apo-TDH, which is consistent with the absence of the co-factor or an inhibitor in our structure. Interestingly, there is some elec-

IUCr macros version 2.1.11: 2020/04/29 
tron density for the closed conformation of this loop in the vicinity of Thr 183 suggesting an alternative location for the preceding glycine residue (Gly 182).

Acknowledgements. We are indebted to Diamond Light Source (DLS, UK) for provision of synchrotron beam time and travel support. AA was supported by an Impact Studentship from the Division of Medicine at UCL.

\section{References}

Adjogatse, E., Erskine, P., Wells, S. A., Kelly, J. M., Wilden, J. D., Chan, A. W. E., Selwood, D., Coker, A., Wood, S. \& Cooper, J. B. (2018). Acta Cryst. D 74, 861-876. https://doi.org/10.1107/S2059798318009208.

Banawas, S. S. (2018). Biomed. Res. Int. doi: 10.1155/2018/8414257.

Barton, G. J. (1993). Prot. Eng. 6, 37-40.

Battye, T. G. G., Kontogiannis, L., Johnson, O., Powell, H. R. and Leslie, A. G. W. (2011) Acta Crystallogr. D 67, 271-281.

Bird, M. I. \& Nunn, P. B. (1983). Biochem. J. 214, 687-694.

Bowyer, A., Mikolajek, H., Stuart, J. W., Wood, S. P., Jamil, F., Rashid, N., Akhtar, M. \& Cooper, J. B. (2009). J. Struct. Biol. 168, 294-304.

Boylan, S. A. \& Dekker, E. E. (1978). Biochem. Biophys. Res. Commun. 85, 190-197.

Boylan, S. A. \& Dekker, E. E. (1981). J. Biol. Chem. 256, 1809-1815.

Brünger, A. T., Adams, P. D., Clore, G. M., DeLano, W. L., Gros, P., Grosse-Kunstleve, R. W., Jiang, J. S., Kuszewski, J., Nilges, M., Pannu, N. S., Read, R. J., Rice, L. M., Simonson, T. \& Warren, G. L. (1998). Acta crystallogr. D 54, 905-921.

Casañal, A., Lohkamp, B. \& Emsley, P. (2020). Prot. Sci. 29, 1069-1078.

Cross, G. A., Klein, R. A. \& Linstead, D. J. (1975). Parasitol. 71, 311-326.

Edgar, A. J. (2002). BMC Genet. 3, 18.

Edgar, A. J. (2005). BMC Genomics 6, 32.

Emsley, P., Lohkamp, B., Scott, W. G. \& Cowtan, K. (2010). Acta Crystallogr. D 66, 486-501.

Evans, P. R. \& Murshudov, G. N. (2013). Acta Crystallogr. D 69, 1204-1214.

IUCr macros version 2.1.11: 2020/04/29 
Fonknechten, N., Chaussonnerie, S., Tricot, S., Lajus, A., Andreesen, J. R., Perchat, N., Pelletier, E., Gouyvenoux, M., Barbe, V., Salanoubat, M., Le Paslier, D., Weissenbach, J., Cohen, G. N., \& Kreimeyer, A. (2010). BMC genomics 11, 555.

Higashi, N., Matsuura, T., Nakagawa, A. \& Ishikawa, K. (2005). Acta Cryst. F 61, 432-434.

Ishikawa, K., Higashi, N., Nakamura, T., Matsuura, T. \& Nakagawa, A. (2007). J. Mol. Biol. 366, 857-867.

Kantardjieff, K. A. \& Rupp, B. (2003). Prot. Sci. 12, 1865-1871.

Karplus, P. A. \& Diederichs, K. (2012). Science 336, 1030-1033.

Kavanagh, K. L., Jörnvall, H., Persson, B. \& Oppermann, U. (2008). Cell. Mol. Life Sci. 65, 3895-3906.

Krissinel, E. \& Henrick, K. (2007). J. Molec. Biol. 372, 774-797.

Lam, V. M. S., Chan, I. P. R. \& Yeung, Y. G. (1980). J. Gen. Microbiol. 117, 539-542.

Linstead, D. J., Klein, R. A. \& Cross, G. A. M. (1977). J. Gen. Microbiol. 101, 243-251.

Machielsen, R. \& van der Oost, J. (2006). FEBS J. 273, 2722-2729.

Marcus, J. P. \& Dekker, E. E. (1993). J. Bacteriol. 175, 6505-6511.

Mazet, M., Morand, P., Biran, M., Bouyssou, G., Courtois, P., Daulouède, S., Millerioux, Y., Franconi, J.-M., Vincendeau, P., Moreau, P. \& Bringaud, F. (2013). PLoS Negl. Trop. Dis. 7, e2587.

Millerioux, Y., Ebikeme, C., Biran, M., Morand, P., Bouyssou, G., Vincent, I. M., Mazet, M., Riviere, L., Franconi, J.-M., Burchmore, R. J. S., Moreau, P., Barrett, M. P. \& Bringaud, F. (2013). Mol. Microbiol. 90, 114-129.

Murshudov, G. N., Vagin, A. A. \& Dodson, E. J. (1997). Acta Crystallogr. D 53, 240-255.

Murshudov, G. N., Skubak, P., Lebedev, A. A., Pannu, N. S., Steiner, R. A., Nicholls, R. A., Winn, M. D., Long, F. \& Vagin, A. A. (2011). Acta Crystallogr. D 67, 355-367.

Neumann-Schaal, M., Jahn, D. \& Schmidt-Hohagen, K. (2019). Frontiers Microbiol. doi: 10.3389/fmicb.2019.00219.

Ritter, B., Denisov, A. Y., Philie, J., Deprez. C., Tung, E. C., Gehring, K. \& McPherson, P. S. (2004). EMBO J. 23, 3701-3710.

Schmidt, A., Sivaraman, J., Li, Y., Larocque, R., Barbosa, J. A. R. G., Smith, C., Matte, A., Schrag, J. D. \& Cygler, M. (2001). Biochemistry 40, 5151-5160.

Ueatrongchit, T. \& Asano, Y. (2011). Anal. Biochem. 410, 44-56.

IUCr macros version 2.1.11: 2020/04/29 
Vagin, A., \& Teplyakov, A. (2010). Acta crystallograph. $\quad$ D $\quad \mathbf{6 6}, \quad 22-25$ https://doi.org/10.1107/S0907444909042589.

Winn, M. D., Ballard, C. C., Cowtan, K. D., Dodson, E. J., Emsley, P., Evans, P. R., Keegan, R. M., Krissinel, E. B., Leslie, A. G. W., McCoy, A., McNicholas, S. J., Murshudov, G. N., Pannu, N. S., Potterton, E. A., Powell, H. R., Read, R. J., Vagin, A. \& Wilson, K. S. (2011). Acta Crystallogr. D 67, 235-242.

Yoneda, K., Sakuraba, H., Araki, T. \& Ohshima, T. (2012). J. Biol. Chem. 287, 12966-12974.

Yoneda, K., Sakuraba, H., Muraoka, I., Oikawa, T. \& Ohshima, T. (2010). FEBS J. 277, $5124-5132$. 
Table 1. X-ray data collection, processing and refinement statistics. The values in parentheses refer to the outer resolution shell.

\begin{tabular}{|c|c|}
\hline Beamline & DLS I04-1 \\
\hline Wavelength $(\AA)$ & 0.92 \\
\hline Space group & $P 3_{1} 21$ \\
\hline$a, b(\AA)$ & 180.87 \\
\hline$c(\AA)$ & 88.35 \\
\hline$\alpha, \beta, \gamma\left({ }^{\circ}\right)$ & $90,90,120$ \\
\hline Solvent content (\%) & 78.9 \\
\hline$N^{\circ}$ molecules per asymmetric unit & 2 \\
\hline Matthews coefficient $\left(\AA^{3} \mathrm{Da}^{-1}\right)$ & $\overline{5.8}$ \\
\hline Mosaic spread $\left({ }^{\circ}\right)$ & 0.36 \\
\hline Resolution $(\AA)$ & $63.2-2.6(2.7-2.6)$ \\
\hline${ }^{*} R_{\text {merge }}(\%)$ & $11.8(177.8)$ \\
\hline$\# R_{\text {meas }}(\%)$ & $12.5(189.7)$ \\
\hline${ }^{\$} \mathrm{CC}_{1 / 2}(\%)$ & $99.8(52.6)$ \\
\hline Average $\mathrm{I} / \sigma(\mathrm{I})$ & $12.2(1.1)$ \\
\hline Multiplicity & $9.3(8.3)$ \\
\hline$N^{\circ}$ observed reflections & $498,386(64,046)$ \\
\hline$N^{\circ}$ unique reflections & $53,475(7,758)$ \\
\hline Wilson plot $B$-factor $\left(\check{\AA}^{2}\right)$ & 61.4 \\
\hline R-factor (\%) & 20.0 \\
\hline Free R-factor (\%) & 23.9 \\
\hline$N^{\mathrm{O}}$ reflections in work/test sets & $49,943 / 2,500$ \\
\hline RMSD bond lengths $(\AA)$ & 0.0094 \\
\hline RMSD bond angles $\left({ }^{\circ}\right)$ & 1.790 \\
\hline Mean protein $B$-factor $\left(\AA^{2}\right)$ & 80.6 \\
\hline
\end{tabular}

${ }^{*} R_{\text {merge }}=\Sigma_{h k l} \Sigma_{i}\left|I_{i}(h k l)-<I(h k l)>\right| / \Sigma_{h k l} \Sigma_{i} I_{i}(h k l)$

${ }^{\#} R_{\text {meas }}=\Sigma_{h k l}\{N(h k l) /[N(h k l)-1]\}^{1 / 2} \Sigma_{i}\left|I_{i}(h k l)-<I(h k l)>\right| / \Sigma_{h k l} \Sigma_{i} I_{i}(h k l)$

$\$$ Half-set correlation coefficient (Karplus \& Diederichs, 2012). 
2-Amino-3-ketobutyrate<smiles>CC(O)C([NH3+])C(=O)[O-]</smiles>

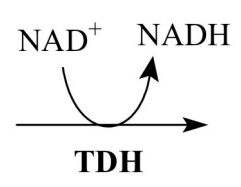<smiles>CC(=O)C([NH3+])C(=O)[O-]</smiles>

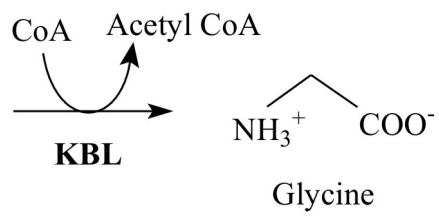

L-Threonine

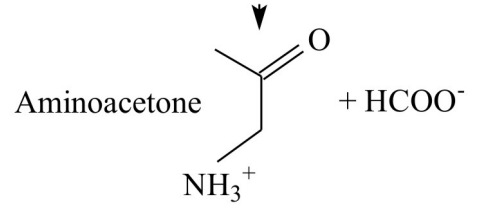

Fig. 1. The reaction catalysed by L-threonine dehydrogenase.

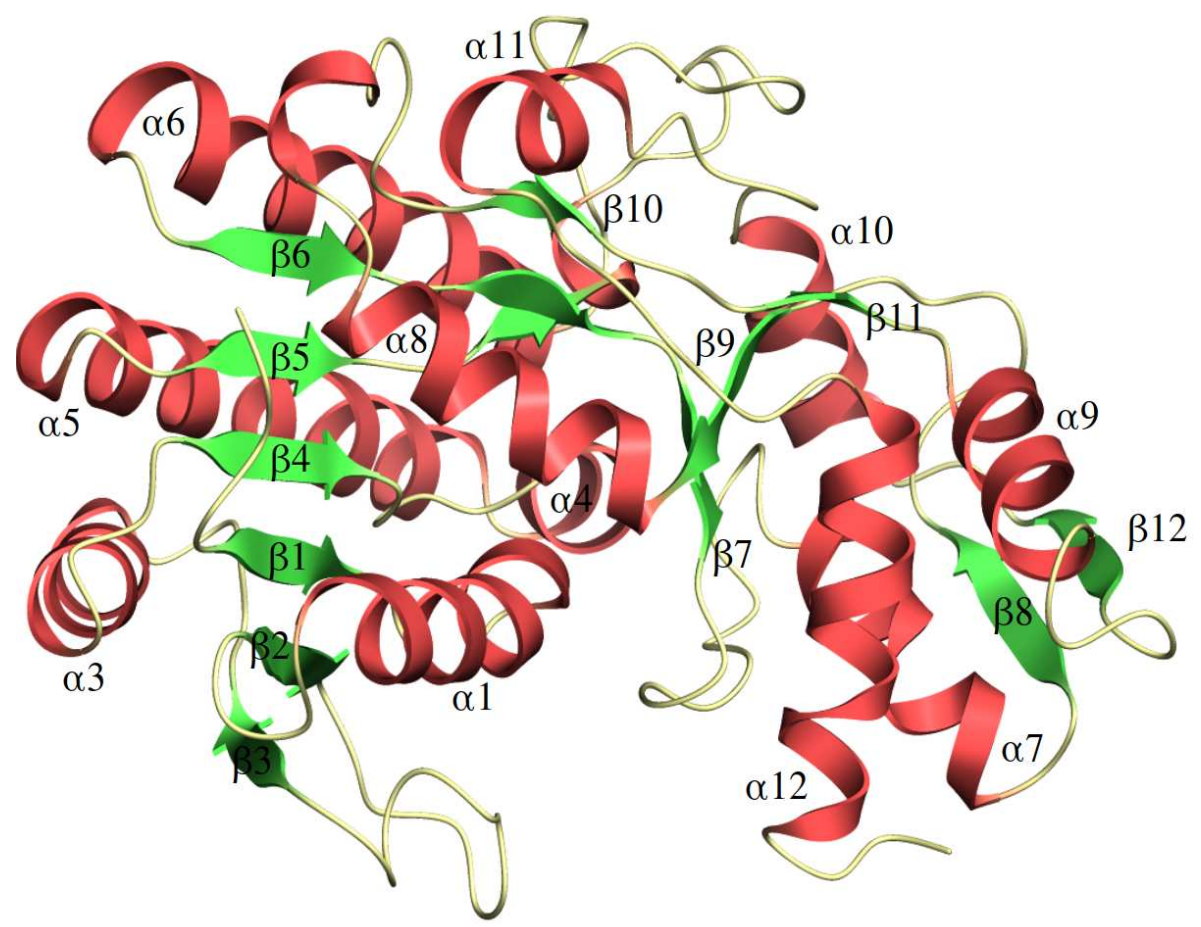

Fig. 2. The 3D structure of L-threonine dehydrogenase from $C$. difficile at 2.6 A resolution. 


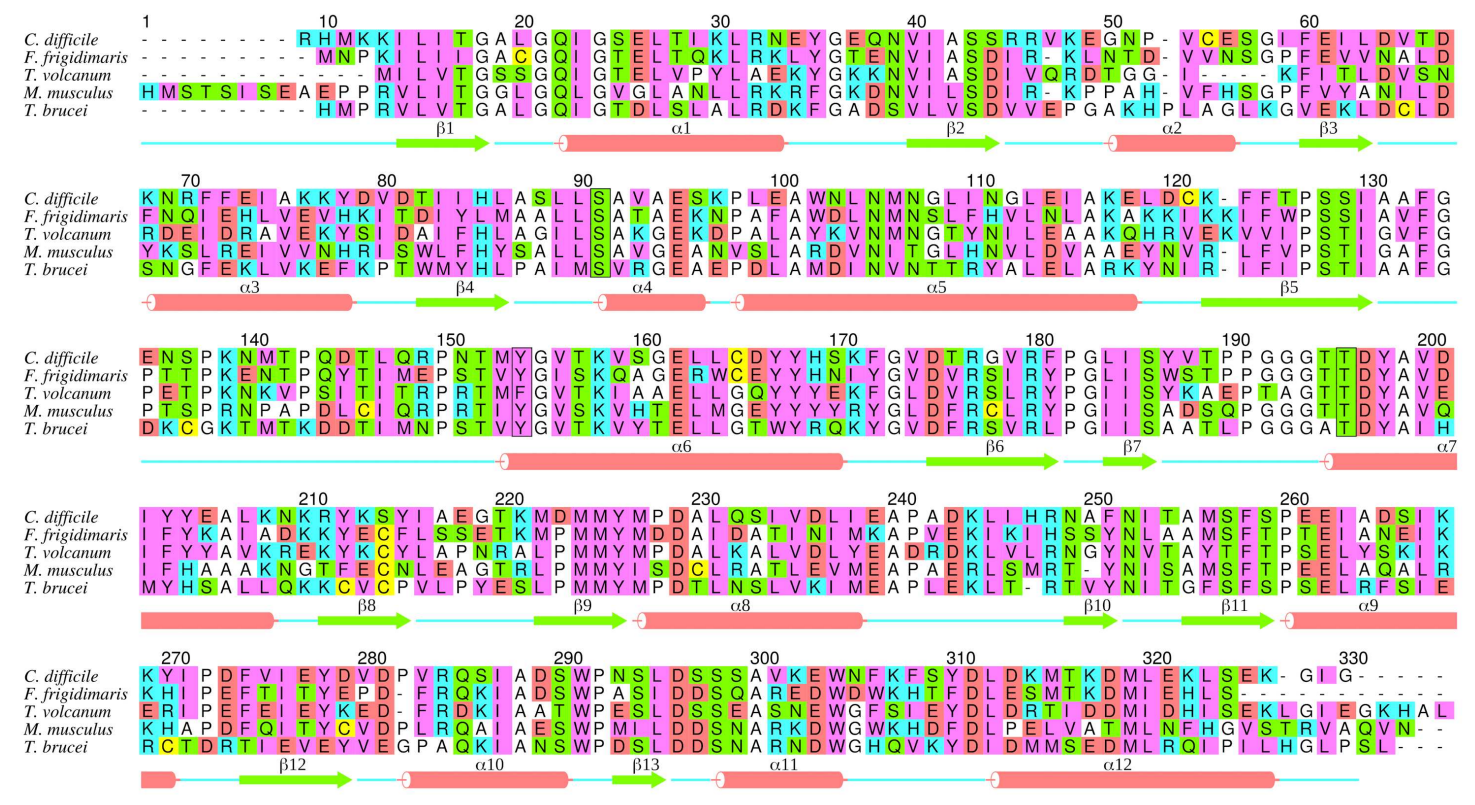

Fig. 3. A sequence alignment of $C$. difficile TDH with the enzymes from $F$. frigidimaris, T. volcanum, mouse (M. musculus) and T. brucei. The amino acids are coloured according to the scheme: acidic: red, basic: pale-blue, neutral-polar: green, hydrophobic: purple, cysteine: yellow and the structurally important residues Gly, Ala, Pro: white. The numbers shown correspond to the combined alignment. The residues involved in substrate and inhibitor binding in related TDH's (Ser 80, Tyr 142 and Thr 184, in C. difficile numbering) are shown boxed at positions 91, 154 and 196. Note that the tyrosine is not invariant. The secondary structure elements of the protein are labelled and displayed with a similar colour scheme to Fig. 2. This figure was prepared using Alscript (Barton, 1993). 


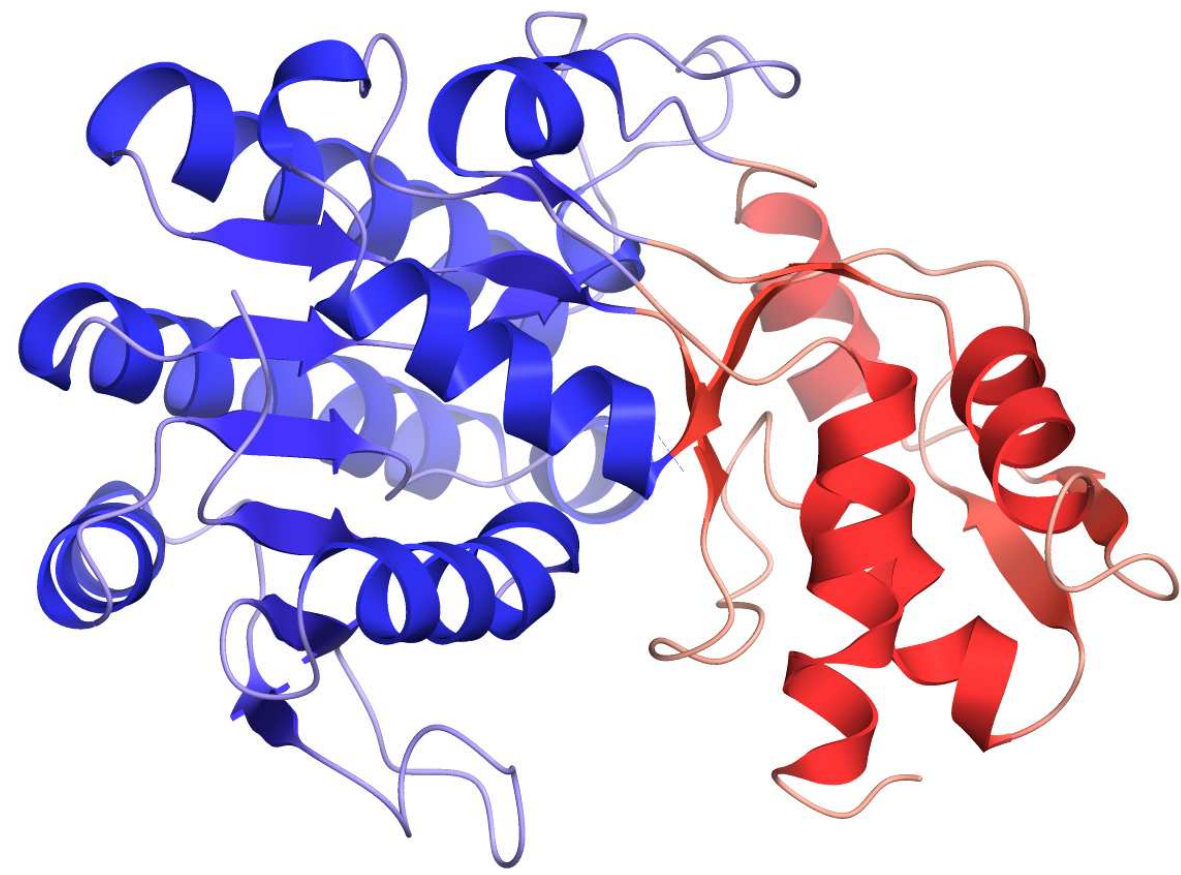

Fig. 4. The two domains of TDH. The Rossmann fold domain which is formed predominantly by the N-terminal portion of the protein is coloured blue and the C-terminal catalytic domain is shown red. 


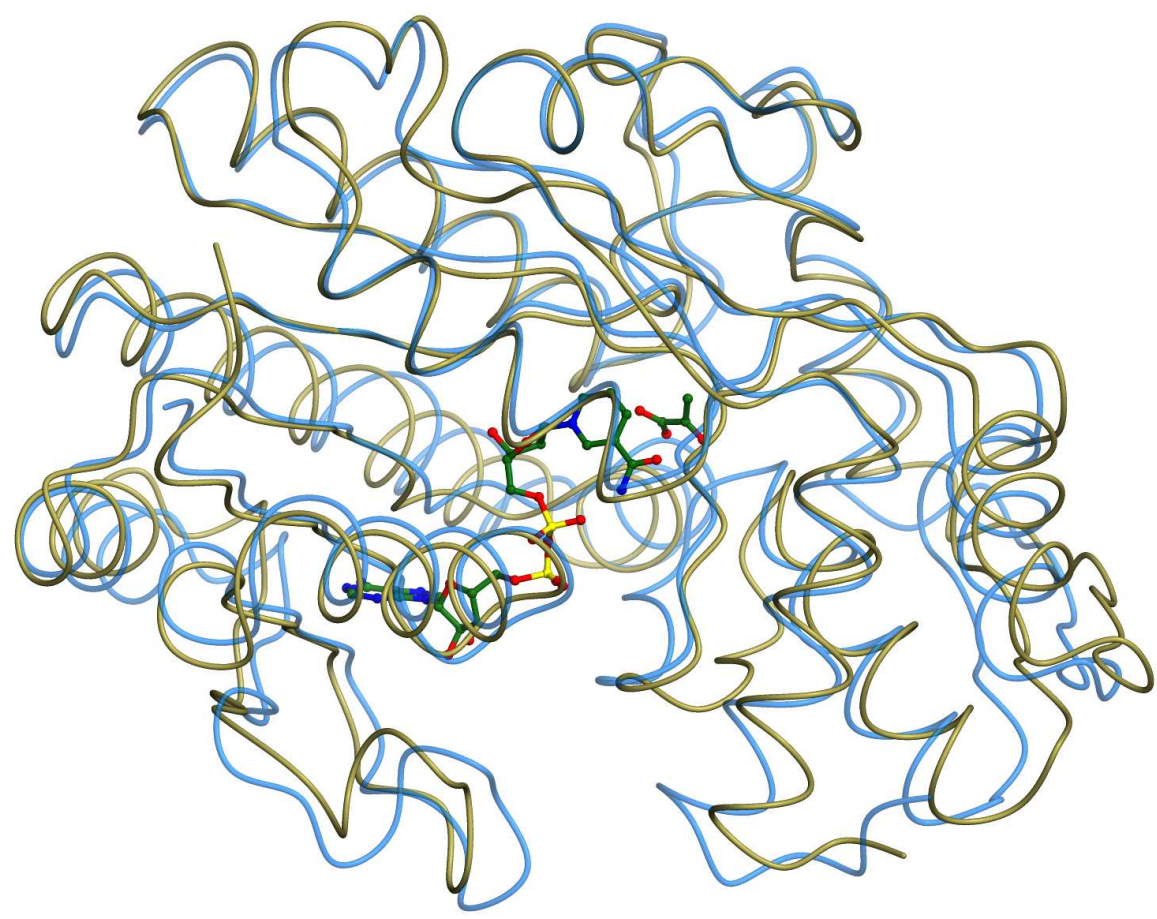

Fig. 5. A superposition of $C$. difficile TDH (beige) with the T. brucei enzyme (pale blue). The NAD cofactor and the competitive inhibitor pyruvate in the T. brucei complex (RCSB-ID: 5lc1) are shown in ball-and-stick representation to indicate the position of the active site. 


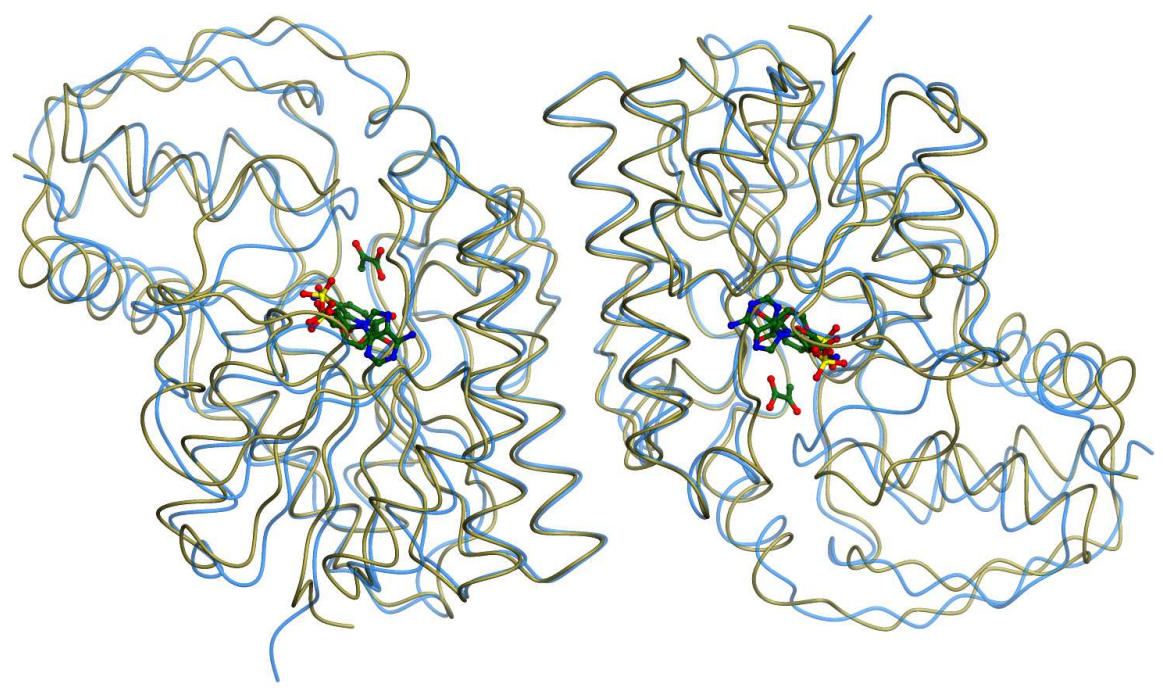

Fig. 6a. A superposition of the $C$. difficile TDH dimer (beige) with that of the $T$. brucei enzyme (pale blue) demonstrates the strong conservation of the dimeric quaternary structure. The NAD cofactor and inhibitor pyruvate bound to the T. brucei enzyme are also shown.

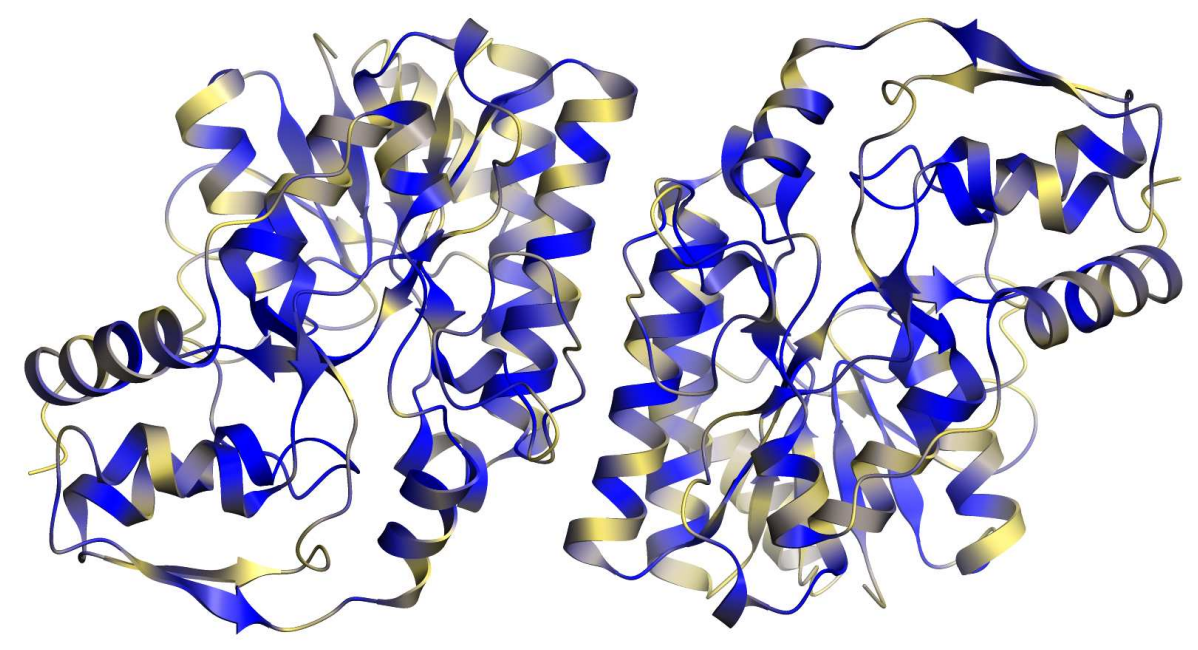

Fig. 6b. The $C$. difficile TDH dimer coloured according to the conservation of the amino acid sequences shown in Fig. 3. Blue indicates the strongly conserved regions while beige indicates the most variable. The sequence conservation in the vicinity of helices which form the dimer interface is apparent. The conservation analysis was done using ProtSkin (Ritter et al., 2004). 


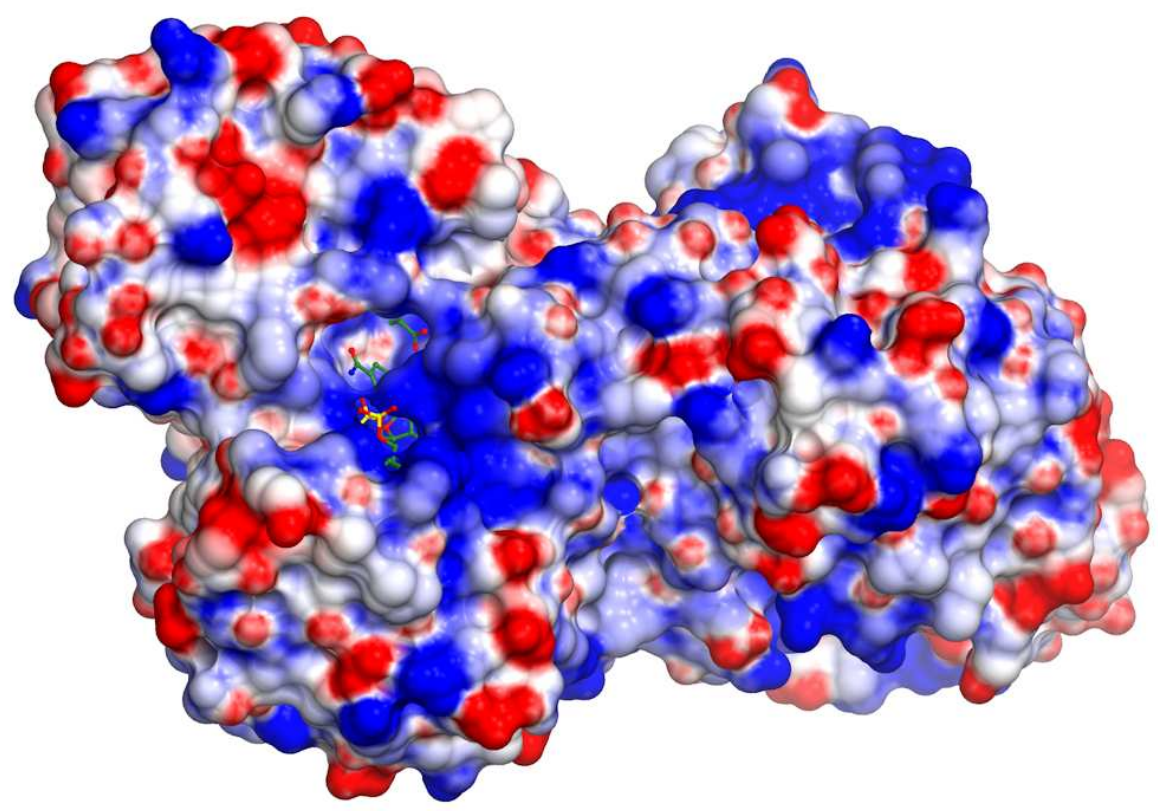

Fig. 6c. The $C$. difficile solvent accessible surface of the $C$. difficile TDH dimer coloured according to electrostatic potential. The basic nature of the NAD binding site is visible for the left-hand monomer (red: acidic, blue: basic). The NAD and pyruvate inhibitor present in the T. bruce $i$ structure are also shown. 


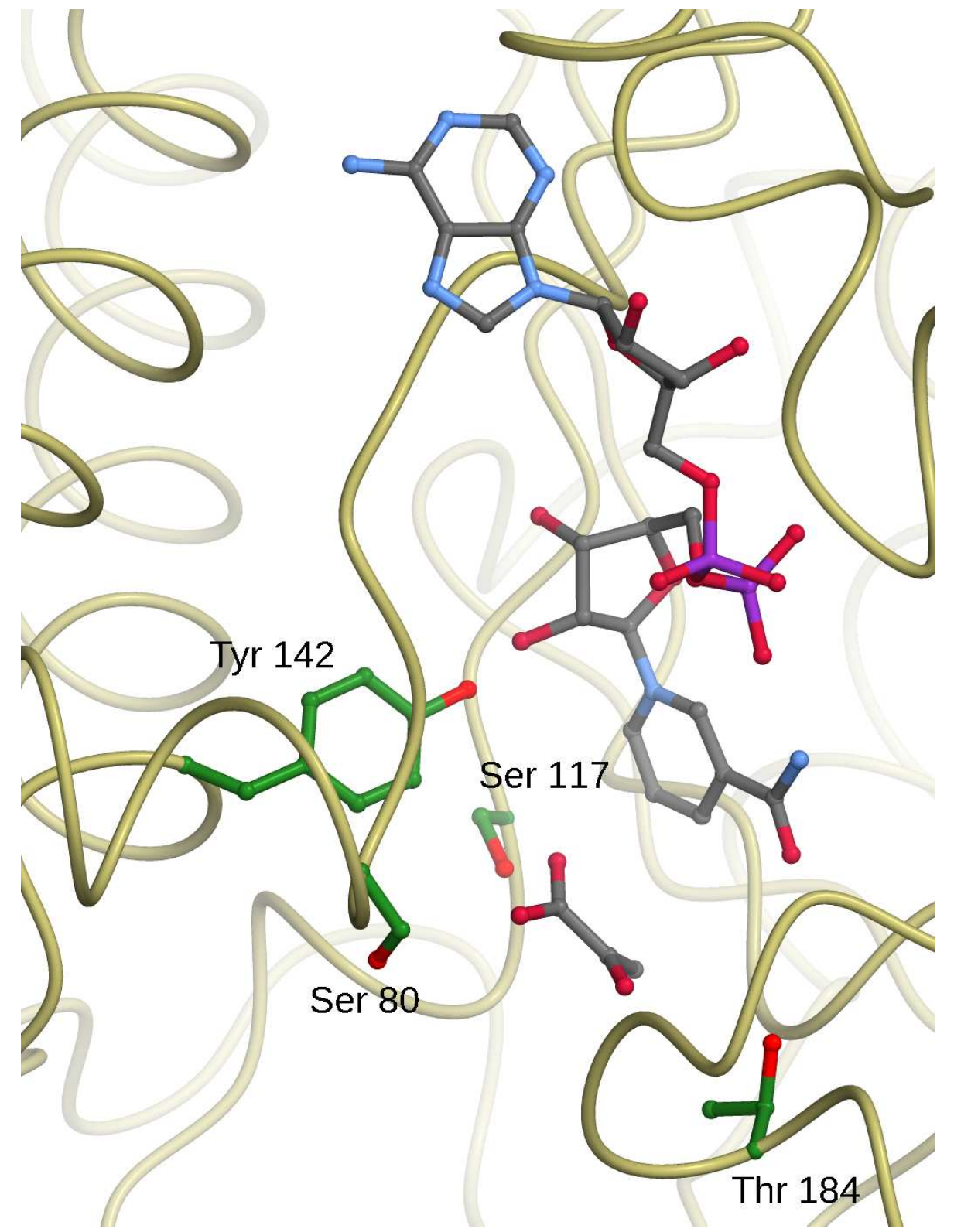

Fig. 7. The active site of $C$. difficile TDH. The side chains of the residues Ser 80, Ser 117, Tyr 142 and Thr 184 which are within hydrogen-bonding distance of the pyruvate present in the T. brucei TDH structure are labelled. The $T$ brucei pyruvate and NAD are shown with grey carbon atoms.

\section{Synopsis}

We have cloned, expressed and crystallised the threonine dehydrogenase from Clostridium difficile and have determined the X-ray structure of the apoenzyme form at $2.6 \AA$ resolution with an R-factor of $20.0 \%$ and an R-free of $23.9 \%$.

IUCr macros version 2.1.11: 2020/04/29 